\title{
ARTICLE Structural insights into secretory immunoglobulin A and its interaction with a pneumococcal adhesin
}

\author{
Yuxin Wang $\mathbb{D}^{1,2}$, Guopeng Wang ${ }^{2}$, Yaxin $\mathrm{Li}^{1,2}$, Qinyu Zhu ${ }^{1,2}$, Hao Shen ${ }^{1,2}$, Ning Gao $\mathbb{D}^{2,3,4}$ and Junyu Xiao $\mathbb{D}^{1,2,4}$
}

\begin{abstract}
Secretory Immunoglobulin A ( $\mathrm{Slg} A)$ is the most abundant antibody at the mucosal surface. It possesses two additional subunits besides IgA: the joining chain (J-chain) and secretory component (SC). SC is the ectodomain of the polymeric immunoglobulin receptor (plgR), which functions to transport IgA to the mucosa. How the J-chain and plgR/SC facilitate the assembly and secretion of SIgA remains incompletely understood. Furthermore, during the infection of Streptococcus pneumoniae, the pneumococcal adhesin SpsA hijacks plgR/SC and SIgA to gain entry to human cells and evade host defense. How SpsA targets plgR/SC and SlgA also remains elusive. Here we report a cryo-electron microscopy structure of the Fc region of IgA1 (Fca) in complex with the J-chain and SC (Fca-J-SC), which reveals the organization principle of SIgA. We also present a structure of Fca-J-SC complexed with SpsA, which uncovers the specific interactions between SpsA and human plgR/SC. These results advance the molecular understanding of SlgA and shed light on S. pneumoniae pathogenesis.
\end{abstract}

Cell Research (2020) 30:602-609; https://doi.org/10.1038/s41422-020-0336-3

\section{INTRODUCTION}

The mucous membrane covers $\sim 400 \mathrm{~m}^{2}$ surface of internal organs in the human body. Immunoglobulin $A(\lg A)$ is the most predominant antibody in mucosal secretions. ${ }^{1}$ In contrast to $\lg \mathrm{A}$ in serum that is mostly monomeric, mucosal IgA is mainly present as dimers (dlgA), in which two $\lg A$ molecules are linked together by another protein designated the joining chain (J-chain). ${ }^{2}$ The $\mathrm{J}$ chain is also an integral component of the IgM pentamer (plgM). ${ }^{3}$ The heavy chains of $\lg \mathrm{A}$ and $\operatorname{lgM}$ contain unique C-terminal extensions known as the tailpieces, which are essential for their oligomerization and covalent linkage to the J-chain. ${ }^{4-6}$ Furthermore, an additional polypeptide called the secretory component (SC) is present in mucosal $\lg \mathrm{A}$ and $\lg \mathrm{M}$, and such $\lg \mathrm{A}$ and $\lg \mathrm{M}$ complexes are often referred to as secretory $\lg A$ and $\lg M$ (SIgA and SIgM). SC is the ectodomain of the polymeric immunoglobulin receptor (plgR), which functions to transport dlgA and plgM through the mucosal epithelial cells. ${ }^{7-10}$ SlgA forms a critical first line of defense against pathogens at the mucosal surface, and also likely plays an important role in regulating the homeostasis of microbiota. ${ }^{11,12}$ SIgA in breast milk is important for protecting the newborn babies until their own immune systems have developed. Despite the fact that the composition of SIgA and certain details of its assembly process have long been established, the three-dimensional structure of SIgA has remained insufficiently characterized.

Due to the critical function of SIgA in immune defenses, various pathogens have developed strategies to disrupt its function. Streptococcus pneumoniae, also known as pneumococcus, is a Gram-positive bacterium that causes millions of deaths worldwide. $^{13,14}$ It is an opportunistic pathogen residing in the upper respiratory tract of many people especially young children.
In individuals with a weak immune system, the bacterium can invade a wide range of organs including the brain, causing severe diseases such as pneumonia, sepsis, and meningitis. S. pneumoniae SlgA-binding protein ( $\mathrm{SpsA}$; also known as $\mathrm{CbpA}, \mathrm{PspC}$ ) is a pneumococcal adhesin that binds to SlgA. ${ }^{15}$ The binding is mediated by the SC subunit in SIgA, and likely impairs the bacterial clearance function of SlgA. Furthermore, SpsA also interacts with unliganded SC and plgR, and the interaction with plgR may enhance bacterial adherence and facilitate its cellular invasion. ${ }^{16}$ How SpsA selectively recognizes human plgR/SC is unclear.

Here we report the cryo-electron microscopy (cryo-EM) structure of a complex containing the $\mathrm{Fc}$ region of human $\lg \mathrm{A} 1$ (FCa), J-chain, and SC at $3.2 \AA$ resolution. Comparison of this structure with that of $\mathrm{FC} \mu-J-\mathrm{SC}^{17}$ reveals a more complete J-chain and distinctive features for the interactions between Fca, J-chain, and SC. We also investigated the interaction between SIgA and $S$. pneumoniae adhesin SpsA, and determined a cryo-EM structure of human Fca-J-SC in complex with the N-terminal domain (NTD) of SpsA, which shows how human plgR/SC is specifically exploited by SpsA to promote $S$. pneumoniae pathogenesis.

\section{RESULTS}

Structure determination of the Fca-J-SC complex

We co-expressed human Fca with J-chain in HEK293F cells and isolated the Fca-J complex containing the dimeric Fca. SC was individually expressed and purified, and then incubated with the Fca-J sample to form the Fca-J-SC tripartite complex. We then determined its structure at $3.2 \AA$ resolution (as judged by the FSC 0.143 criterion) by the single particle cryo-EM method (Fig. 1;

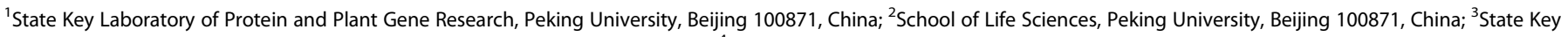
Laboratory of Membrane Biology, Peking University, Beijing 100871, China and ${ }^{4}$ Peking-Tsinghua Center for Life Sciences, Peking University, Beijing 100871, China Correspondence: Junyu Xiao (junyuxiao@pku.edu.cn)

These authors contributed equally: Yuxin Wang, Guopeng Wang, Yaxin Li

Received: 21 February 2020 Accepted: 23 April 2020

Published online: 12 May 2020 


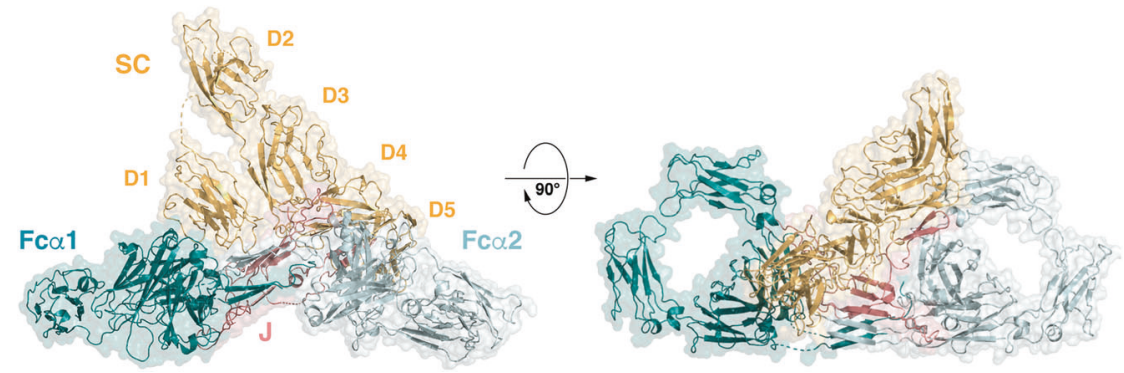

Fig. 1 Cryo-EM structure of the human Fca-J-SC complex. The cryo-EM structure of the human Fc $\alpha$-J-SC complex is shown in two views. The regions corresponding to $\mathrm{Fc} \alpha 1$, Fc $\alpha 2$, J-chain, and SC are shown in teal, light cyan, salmon, and gold, respectively. Similar color schemes are used in all figures unless otherwise indicated. The five immunoglobulin-like domains in SC are indicated as D1-D5.

Supplementary information, Fig. S1). Most regions of the EM map exhibited high enough resolution for unambiguous structural assignment and analyses (Supplementary information, Figs. S1e and S2). The statistics for cryo-EM data collection and processing, as well as structural refinement and validation, are summarized in Supplementary information, Table S1.

Structure of the $\lg A$ dimer and its interaction with the J-chain The two IgA molecules in SlgA are linked via Cys471-mediated disulfide bonds to the J-chain. Earlier EM analyses show that dlgA displays a double-Y-like shape. ${ }^{18}$ Solution scattering studies suggest that the two Fca do not dock to each other in a straight manner in the $\lg \mathrm{A}$ dimer, but adopt a slightly bent end-to-end arrangement. ${ }^{19}$ Consistent with these results, our structure shows that the Fca dimer has a boomerang-like shape (Fig. 2a) and resembles a portion of the plgM structure we determined recently ${ }^{17}$ (Fig. 2b). Each tailpiece of Fca contains a $\beta$-strand, like that of $F_{c} \mu$ in IgM, and four such tailpiece strands bundle together to mediate the interactions between the two Fca molecules. The EM densities are discontinuous between the tailpieces and the rest of heavy chains in Fca1 (Fig. 2a), yet the strands in the tailpieces can still be fully resolved, suggesting that these strands are structurally stable and serve to fasten the IgA dimer.

The Fca dimer is further stabilized by the J-chain. Compared with the Fc $\mu$-J complex, a more complete structure of the J-chain is present in Fca-J, due to more extensive interactions between the Fca dimer and the J-chain. Three intrachain disulfide bridges are seen in the J-chain (Fig. 3a): Cys $12^{J}-$ Cys $100^{J}$ (superscript J indicates J-chain residues), Cys $71^{J}-C y s 91^{J}$, and Cys $108^{J}-C y s 133^{J}$, consistent with previous analyses. ${ }^{20,21}$ Cys $14^{J}$ and Cys68 form a disulfide bond with Cys $471^{\mathrm{FCa} B \mathrm{~B}}$ and Cys $471^{\mathrm{Fca} 1 \mathrm{~A}}$, respectively. The central region of the J-chain contains four $\beta$-strands $(\beta 1-\beta 4)$ that interact with the Fca tailpieces (Fig. 3a). Strands $\beta 1-\beta 3$ pack onto the two tailpiece strands of Fca2 to assemble into a $\beta$-sheet, with main chain hydrogen bonds formed between adjacent strands; whereas $\beta 4$ packs onto the tailpieces of Fca1. Robust hydrophobic interactions are present between the two $\beta$-sheets, mediated by Fca residues Val460, Val462, and Met464, and J-chain residues Ile37 ${ }^{J}, \| l e 39^{J}, V^{\prime}$ Val41 ${ }^{J}$, Phe60', and Tyr62 ${ }^{J}$ (Fig. 3b). The $\beta 2-\beta 3$ loop, $\beta 3-\beta 4$ loop, $\beta 5-\beta 6$ hairpin, and the long $C$-terminal hairpin of the J-chain function as four lassos to further interact with the Fca dimer. The $\beta 2-\beta 3$ and $\beta 3-\beta 4$ loops contact the Ca3-tailpiece junctions of $\mathrm{Fca} 2 \mathrm{~B}$ and $\mathrm{Fca} 1 \mathrm{~A}$, respectively (Supplementary information, Fig. S3); whereas the $\beta 5-\beta 6$ and $C$-terminal hairpins form extensive interactions with the $\mathrm{Ca} 2-\mathrm{Ca} 3$ junctions. The $\beta 5-\beta 6$ hairpin, disordered in the $\mathrm{F} C \mu-J$ complex (Fig. $2 \mathrm{~b}$ ), interacts with the $\mathrm{Ca} 2-\mathrm{Ca} 3$ junction of Fca2B via two hydrophobic centers (Fig. 3c): the first involves J-chain residues Val76 ${ }^{J}$, Leu78, and

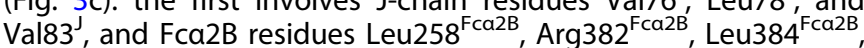
Met433 ${ }^{\mathrm{Fca} 2 \mathrm{~B}}$, and Phe443 ${ }^{\mathrm{Fca2B}}$; and the second involves Thr $86^{j}$, Leu $439^{\mathrm{Fca} 2 \mathrm{~B}}$, Pro $440^{\mathrm{Fca} 2 \mathrm{~B}}$, and Leu $441^{\mathrm{Fca} 2 \mathrm{~B}}$. The C-terminal hairpin interacts with almost the same group of residues in Fca1A
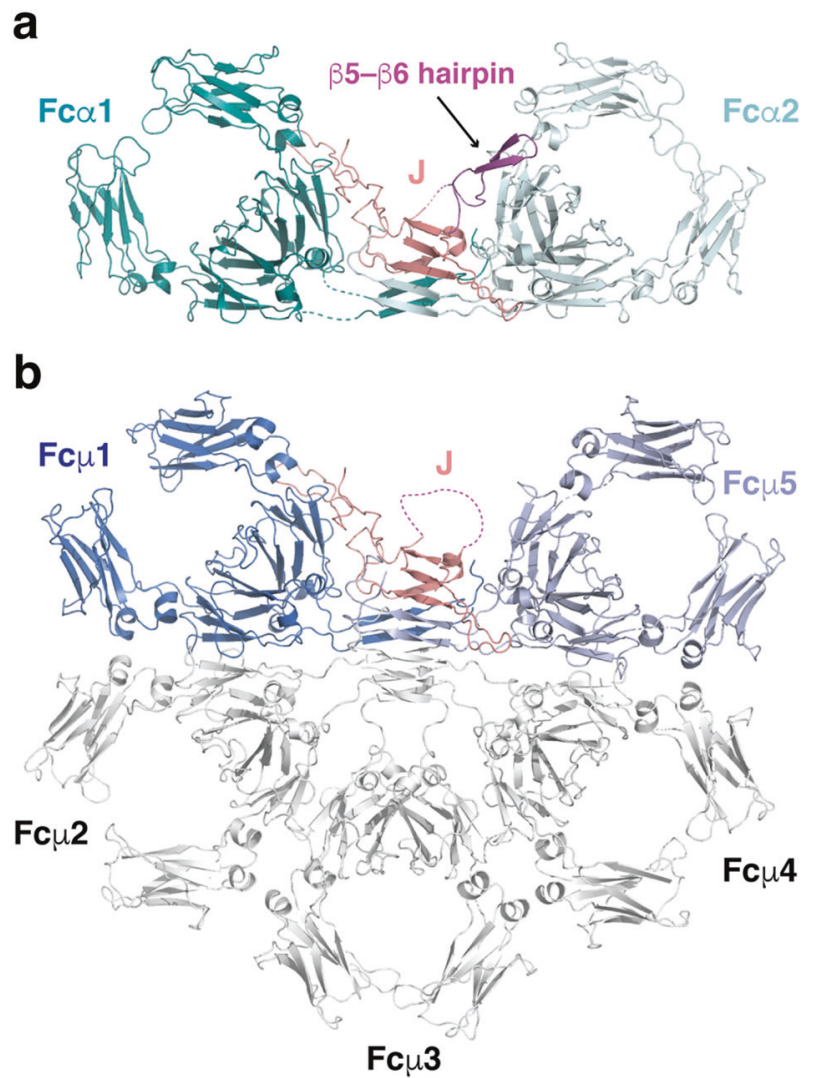

Fig. 2 The core structures of dlgA and plgM. a Overall structure of the dimeric Fc $\alpha$ in complex with the J-chain. The linkers between the tailpieces and the rest of $F c \alpha 1$ heavy chains are indicated with dashed lines, as the EM densities are discontinuous at these regions. The $\beta 5-\beta 6$ hairpin of the J-chain (disordered in the $\mathrm{FC} \mu$-J structure) is highlighted in magenta. b Structure of the pentameric Fc $\mu$ in complex with the J-chain (PDB ID: $6 \mathrm{KXS}$ ). $\mathrm{Fc} 11$ and $\mathrm{F} c \mu 5$ are shown in two shades of blue, whereas Fc $\mu 2-4$ are shown in white. The $\beta 5-$ $\beta 6$ region of the J-chain is disordered in this structure and indicated with a dashed line.

(Fig. 3d). The way that the C-terminal hairpin interacts with Fca1 highly resembles how it binds to $\mathrm{Fc} \mu 1$ in plgM (Fig. 2).

Interaction between dlgA and SC

Extensive biochemical and biophysical studies have established that plgR/SC forms bidentate interactions with dlgA, with both its D1 and D5 domains involved. ${ }^{22-28}$ This is borne out by the cryoEM structure (Fig. 4a). The D1 domain of plgR/SC binds to Fca-J using its three complementarity-determining regions (CDR)-like loops (Fig. 4b), and the molecular interactions are in many ways 


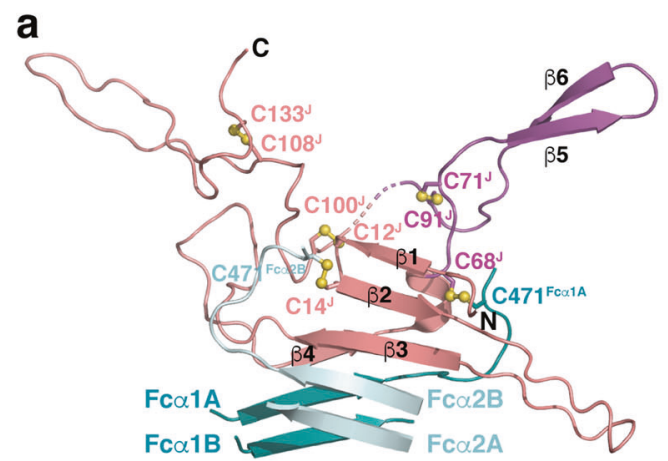

C
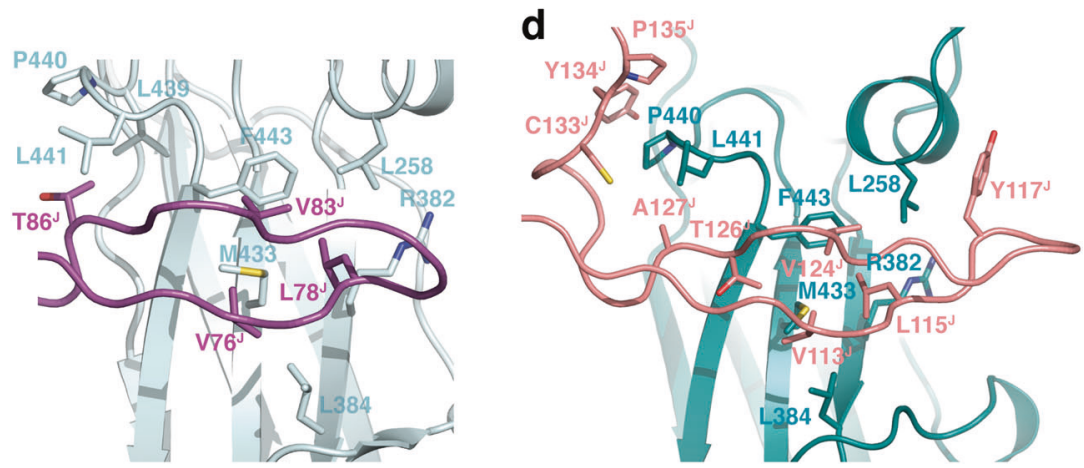

Fig. 3 The interactions between the J-chain and Fca. a Overall structure of the J-chain. The sulfur atoms in Cys are depicted as orange spheres. The $\beta$-strands in the J-chain and Fc $\alpha$ tailpieces are indicated. $\mathbf{b}$ Interactions between two J-chain strands and the Fc $\alpha$ tailpieces. c Interactions between the $\beta 5-\beta 6$ hairpin of the J-chain and the C $\alpha 2-C \alpha 3$ junction of Fc $\alpha 2 B$. $\mathbf{d}$ Interactions between the $\mathrm{C}$-terminal hairpin of the J-chain and the $\mathrm{C} \alpha 2-\mathrm{C} \alpha 3$ junction of $F c \alpha 1 A$.

similar to the interactions seen in the FC $\mu$-J-SC complex. ${ }^{17}$ CDR1 mainly contacts the J-chain. Val29 ${ }^{\mathrm{SC}}$ (superscript SC indicates plgR/ $\mathrm{SC}$ residues) is positioned in a pocket formed by J-chain residues Arg105, Asn106, and Ala132 ${ }^{J}$ (Fig. 4C). Asn30 ${ }^{\text {SC }}$ coordinates Arg105 $5^{\prime}$. Arg3 $31^{\text {SC }^{\prime}}$ interacts with Asp $136^{J}$. His32 $2^{\text {SC }}$ packs against Tyr134 ${ }^{J}$. Besides interacting with the J-chain, plgR/SC also directly contacts the Fca molecules at several places. For example, Arg34 ${ }^{\text {SC }}$ in CDR1 forms a salt bridge with Glu363 ${ }^{\mathrm{Fca} 1 \mathrm{~B}}$, which also packs on Tyr55 $5^{\text {SC }}$ in CDR2 (Fig. 4d). Glu53 ${ }^{\text {SC }}$ in CDR2 engages Arg346 3 Fca1A

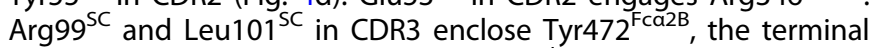
residue of Fca2B, together with $A r g 105^{J}$ (Fig. 4C). To verify the functional relevance of these molecular interactions, we tested the interaction between Fca-J and two SC mutants, V29N/R31S and R99N/L101T. These mutants were designed to bring bulky $\mathrm{N}$-linked glycans into amino acid positions 29 and 99, respectively, which would hinder molecular interactions at CDR1 and CDR3. We previously showed that these mutants displayed greatly reduced interactions with the Fc $\mu$-J complex. ${ }^{17}$ Likewise, they failed to bind Fca-J (Fig. 4e).

The interaction between $\operatorname{dlgA}$ and $\mathrm{plgR} / \mathrm{SC}$ also uniquely involves the D5 domain of plgR/SC, and a disulfide bond is formed between Cys468 ${ }^{\mathrm{SC}}$ and Cys311 in the Ca2 domain of $\mathrm{Fca}^{23}$ In the two previously determined structures of $\mathrm{FCa}_{1}^{29,30}$ Cys311 is present in a hydrophobic pocket and not exposed (Fig. 4f). By contrast, Cys311 $1^{\text {Fca2B }}$ flips out in the Fca-J-SC structure and is located in close proximity to Cys $468^{\mathrm{SC}}$. The EM density did not allow us to model a disulfide bond between them definitively (Supplementary information, Fig. S4a). A nonreducing gel suggests that our Fca-J-SC sample is likely heterogeneous, with disulfide bonds formed between some Fca and SC molecules (Supplementary information, Fig. S4b). Therefore, the EM density at this region is likely derived from the average of disulfide-linked and non-disulfide-linked Fca-SC. For this study, we produced Fca-J and SC separately, incubated them on ice for $1 \mathrm{~h}$, and then isolated the tripartite complex using size exclusion chromatography (see Materials and methods). It is likely that our experimental procedure did not allow sufficient time for the oxidation reaction to fully occur. It is also likely that the formation of this disulfide bond might be facilitated by a protein disulfide isomerase in vivo. In any event, mutation of C468 ${ }^{\mathrm{SC}}$ only slightly decreased the binding between Fca-J and SC in solution (Fig. 4e). This is consistent with previous analyses showing that the initial and primary association of $\operatorname{dlgA}$ with plgR/SC is mediated by interactions at the D1 domain. Disulfide formation between dlgA and Cys $468^{\mathrm{SC}}$ in D5 takes place at a later stage of transcytosis, and the mucosal transport of $\operatorname{dlgA}$ can proceed in its absence. ${ }^{31}$ The main function of this disulfide bond is presumably to increase the stability of SlgA at the mucosal surface and in external fluids.

Interaction between SC and S. pneumoniae SpsA

SpsA comprises a C-terminal phosphorylcholine-binding domain that interacts with pneumococcal cell wall to facilitate bacterial colonization, and an NTD that recruits host proteins including plgR/SC and SIgA. ${ }^{15,32}$ SpsA $^{\text {NTD }}$ contains repeats of the leucine zipper motifs termed R1 and R2, each adopting a three-helix bundle structure. ${ }^{33}$ The YRNYPT hexapeptide involved in binding to $\mathrm{plgR} / \mathrm{SC}$ is located in the loop between helices $\alpha 1$ and $\alpha 2$ in the R1 motif of S. pneumoniae strain ATCC33400 SpsA, where it was first identified. ${ }^{15}$ In many other S. pneumoniae strains, a similar hexapeptide is also present in R2 (Supplementary information, Fig. S5a). To reveal the molecular mechanism underlying the specific recognition of SIgA by SpsA, we produced S. pneumoniae strain ATCC33400 SpsA ${ }^{\text {NTD }}$, reconstituted a Fca-J-SC-SpsA ${ }^{\text {NTD }}$ quadruple complex, and then determined the cryo-EM structure at an overall resolution of $3.3 \AA$ (Fig. 5a, b; Supplementary information, Figs. S1 and S6 and Table S1). The a1-a2 loop of $S p s A^{\text {NTD }}$, especially the YRNYPT hexapeptide, displays high-quality densities and can be clearly resolved (Supplementary information, Fig. S6b). 

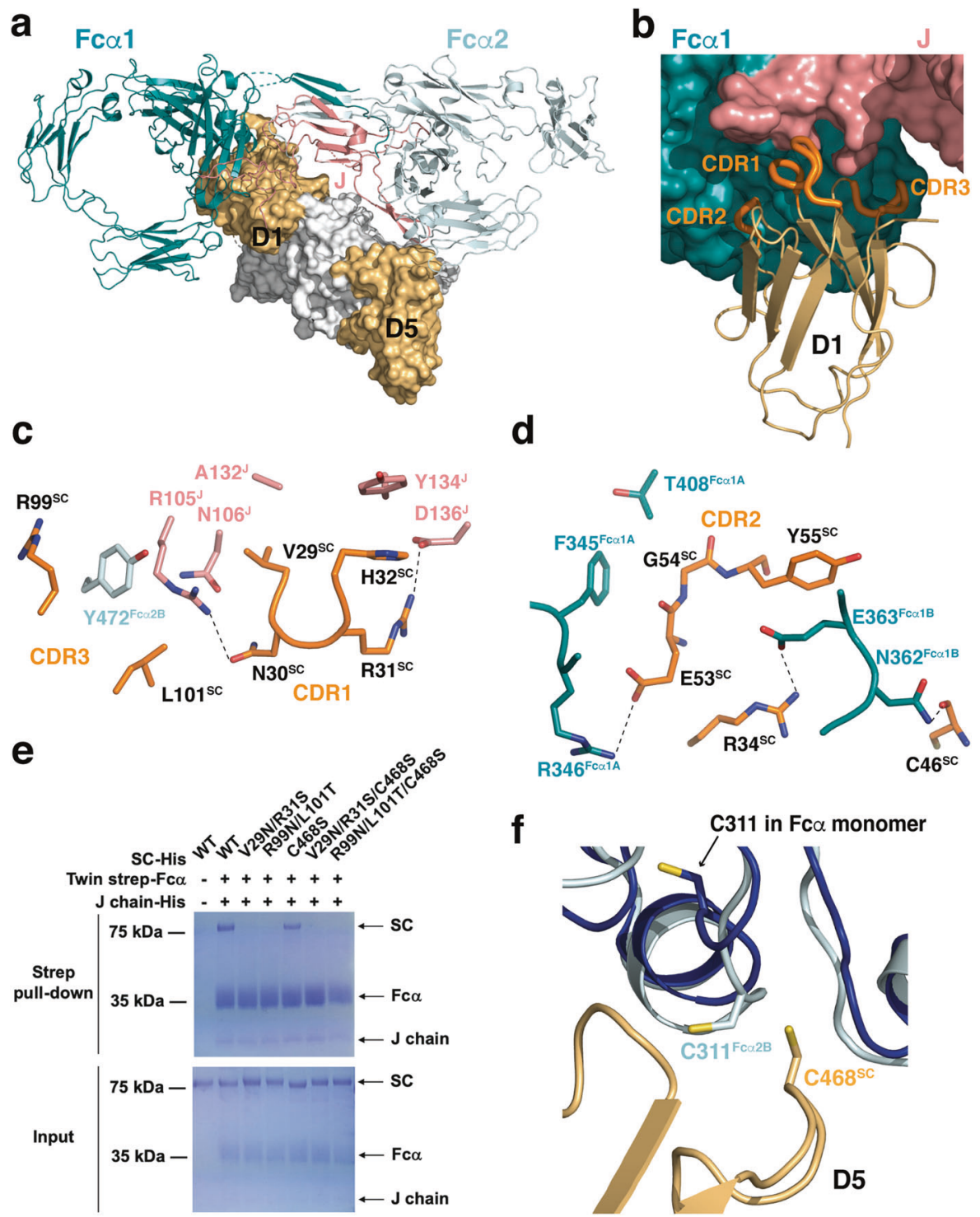

Fig. 4 Interaction between dlgA and SC. a Both the D1 and D5 domains of SC are involved in binding to dlgA. Fc $\alpha$ and the J-chain are shown as ribbon diagrams, whereas SC is shown as a surface representation. The D1 and D5 domains of SC are highlighted in gold. The rest of SC is in white. $\mathbf{b}$ The three CDR-like loops in the D1 domain of SC, highlighted in thicker ribbons, engage Fc 1 and the J-chain. The surfaces of Fc 1 and the J-chain are shown. c Interactions between SC and Fc $\alpha-J$ at the CDR1 and CDR3 regions. Polar interactions are indicated by dashed lines. $\mathbf{d}$ Direct interactions between SC and Fc $\alpha 1$. e SC mutants display reduced interactions with Fc $\alpha-J$. $\mathbf{f}$ The structure of the Fc $\alpha$ monomer (PDB ID: 2QEJ), shown in blue, is overlaid onto Fc $\alpha 2 B$ in the Fc $\alpha$-J-SC structure. Compared with Cys311 in the Fc $\alpha$ monomer, Cys311 ${ }^{\mathrm{Fc} \alpha 2 \mathrm{~B}}$ flips out and can readily form a disulfide bond with Cys $468^{\mathrm{SC}}$.

Sps $A^{\text {NTD }}$ specifically interacts with the D3-D4 domains of human $\mathrm{plgR/SC} .^{34,35}$ In the cryo-EM structure, the a1-a2 loop of SpsA ${ }^{\text {NTD }}$ docks into a pocket at the D3-D4 junction (Fig. 5b,c), formed by the DE loop of D3 and the $C-C^{\prime}$ strands of D4. Notably, this pocket is only present in the ligand-bound conformation of $\mathrm{SC}^{17}$ No significant conformational changes were observed in SC upon Sps $\mathrm{A}^{\mathrm{NTD}}$ binding, except for the $\mathrm{C}-\mathrm{C}^{\prime}$ loop of D4, which tilted slightly to engage the YRNYPT hexapeptide (Supplementary information, Fig. S7a). Tyr198, the first residue in the hexapeptide, forms a hydrogen bond with Tyr365 ${ }^{\text {SC }}$ (Fig. 5d). Arg199 packs on $\operatorname{Trp}_{386}{ }^{\mathrm{SC}}$, and forms a salt bridge with Asp382 ${ }^{\mathrm{SC}}$. Asn200 forms a hydrogen bond with Arg $376^{\text {SC }}$. Tyr201 packs on Pro $283^{\text {SC }}$. Pro202 is surrounded by hydrophobic residues including $\mathrm{Tyr} 365^{\mathrm{SC}}$, Cys $367^{\text {SC }}$, Cys $377^{\text {SC }}$, Leu379 5 , and Leu $424^{\text {SC }}$. Substitution of Tyr201 with an Asp or Pro202 with a Glu abolished the binding of SpsA to SIgA. ${ }^{36}$ Thr203 interacts with Asn $282^{\text {SC }}$. Notably, most of the SC residues described here are specifically present in human (Supplementary information, Fig. S5b), explaining the fact that SpsA only binds to human SIgA. ${ }^{36}$ Other SpsA residues are also involved in interacting with $\mathrm{SC}$ in addition to the YRNYPT hexapeptide, including Tyr206 that forms a hydrogen bond with the main chain carbonyl group of Pro283 $3^{\mathrm{SC}}$, and Arg265 in helix a3 that likely bonds with Asp285 ${ }^{\text {SC }}$ (Fig. 5d). Both of these residues are highly conserved in SpsA (Supplementary information, Fig. S5a).

\section{DISCUSSION}

SlgA is of paramount importance to mucosal immunity. In adults, the daily synthesis of $\lg A$ is greater than all other types of antibody combined, and most of these $\lg A$ molecules are present in mucosal secretions in the form of dimeric SlgA. Despite the long history of SIgA research, its structure has remained elusive until only recently. During the preparation of this manuscript, the 


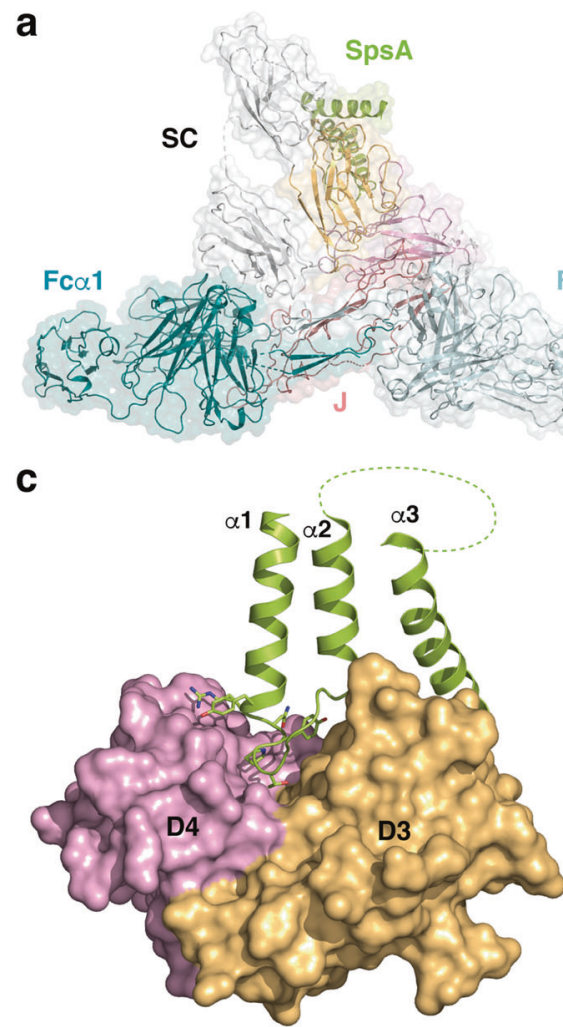

b

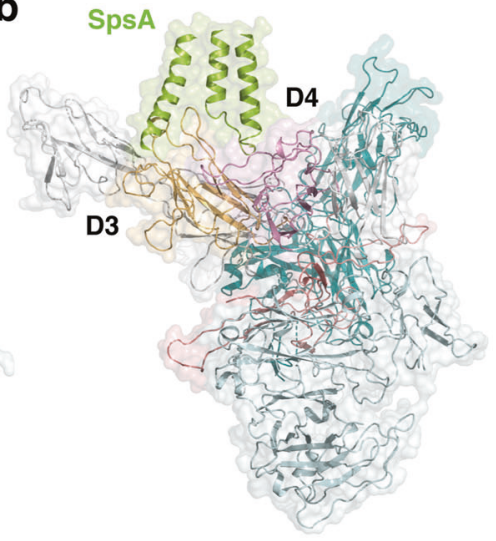

d

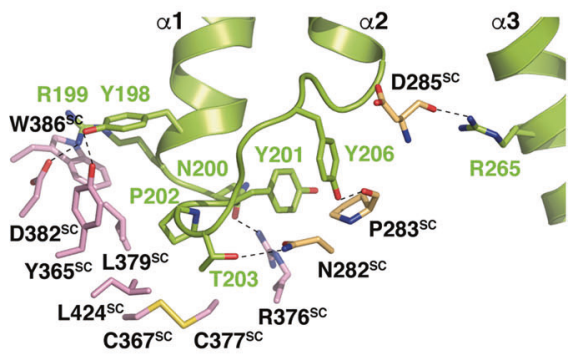

Fig. 5 Interaction between SC and SpsA. a Overall structure of the Fc $\alpha$-J-SC-SpsA ${ }^{\text {NTD }}$ quadruple complex. b Another view of the Fc $\alpha-J-S C$ SpsA ${ }^{\text {NTD }}$ structure. c Structure of the D3-D4 domains of SC in complex with SpsA ${ }^{\text {NTD. }}$. The D3-D4 domains of SC are shown in a surface representation, with D3 and D4 in gold and purple, respectively. SpsA ${ }^{\mathrm{NTD}}$ is shown in lemon, and the side chains of the YRNYPT hexapeptide are depicted. $\mathbf{d}$ Detailed interactions between SC and SpsA.

cryo-EM structures of SIgA have been published by Genentech. ${ }^{37}$ Our Fca-J-SC structure is very similar to the structure reported in this study (Supplementary information, Fig. S7b), since comparison of these two structures reveals a global root mean square deviation of only $1.6 \AA$. The similarity of these independently determined structures demonstrates the sturdiness of the dimeric SlgA core assembly.

$\lg A$ can induce immune signaling by binding to the $\lg A$ specific receptor FcaRI/CD89. ${ }^{38,39}$ However, it is not entirely clear whether monomeric $\lg \mathrm{A}$ and $\mathrm{SIgA}$ can elicit similar immune responses. Crystal structure study reveals a 2:1 FcaRl:Fca complex $^{29}$ (Fig. 6a). In SlgA, only one side of the FcaRl-binding site would be exposed in each Fca (Fig. 6b). The other side is occupied by the J-chain and not available for binding. From a structural point of view, there is no apparent reason to think that SIgA would not bind to FcaRl; nevertheless, it would have to cluster FcaRI molecules in a different arrangement. Whether this altered mode of binding may account for the different immune responses elicited by monomeric $\operatorname{Ig} \mathrm{A}$ and $\mathrm{SIgA}$ remains to be investigated. On the other hand, the J-chain and SC may also contribute to the signaling function of SIgA. For example, it has been shown that the Mac-1 integrin is required as a co-receptor for SIgA to activate immune responses via FcaRl, and the interaction with Mac- 1 is mediated by SC. ${ }^{40,41}$ Recently, human Fc receptor-like 3 (FCRL3) has been identified as a SIgA-specific receptor. ${ }^{42}$ It is likely that the J-chain and/or SC are also involved in the interaction between SIgA and FCRL3.

S. pneumoniae is an important human pathogen. SpsA is a major adhesin of $S$. pneumoniae and plays a role during its infection. SpsA is a modular protein and can bind to both host proteins and the pneumococcal cell wall. Despite the fact that the DNA region encoding SpsA is highly polymorphic, the Y/R-R-N-Y-P-T motif involved in binding to plgR/SC and SlgA is highly conserved, and is present in one or two copies in more than $70 \%$ strains of S. pneumoniae. ${ }^{15,43}$ The unique ability of SpsA to bind plgR/SC and SlgA may contribute to the pathogenesis $S$. pneumoniae in several ways. First, binding to plgR on the nasopharyngeal and lung epithelia as well as brain endothelial cells may facilitate the colonization and internalization of S. pneumoniae. Indeed, SpsAdeficient $S$. pneumoniae showed greatly reduced ability to adhere to, and abolished activity to invade human cells in tissue culture models. ${ }^{16,32}$ Binding to SlgA may also assist in bacterial invasion, since SIgA in complex with its antigens can be internalized by certain cells via reverse transcytosis. ${ }^{44,45}$ On the other hand, the interaction between SpsA and SlgA may impede the defense function of SlgA. By binding to SlgA using its NTD and the bacterial cell wall via its C-terminal domain, SpsA may recruit SlgA to bacterial surface and promote its degradation by S. pneumoniae $\lg \mathrm{A}$ proteases. ${ }^{46}$ Binding of SpsA to SIgA may also sterically hinder SIgA from agglutinating and clearing the bacteria, or block the interaction between SIgA and binding partners to initiate host immune responses.

It is worth noting that SpsA evolves to bind to human plgR/SC specifically, since it does not interact with SIgA and SC from common laboratory animals including mouse, rat, rabbit, and guinea pig. ${ }^{36}$ Indeed, residues in $\mathrm{plgR/SC}$ that participate in the interaction with SpsA are not conserved in these animals (Supplementary information, Fig. S5b). These differences underscore the fact that $S$. pneumoniae is a human-specific pathogen and should be taken into consideration for its study. On the other hand, the unusual capability of SpsA to bind human SC with high selectivity and affinity may allow the development of recombinant SpsA protein as a tool for efficient isolation and purification of human SC, SIgA, and SIgM. 
a

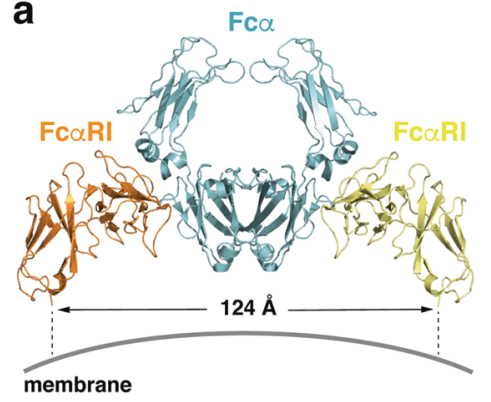

b

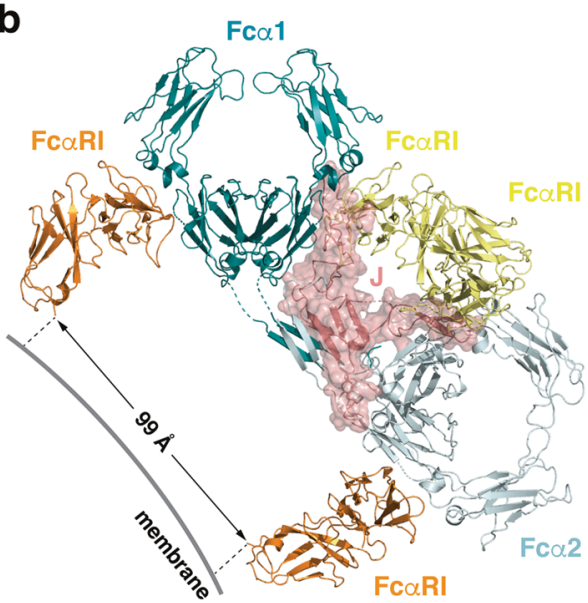

Fig. 6 A hypothetical model of the dlgA-FcaRI complex. a Crystal structure of the 2:1 Fc $\alpha$ RI:Fc $\alpha$ complex (PDB ID: 1OW0). The two Fc $\alpha$ RI molecules are shown in orange and yellow. The distance between the C-terminal ends of the two FcoRI molecules is indicated. $\mathbf{b}$ Two copies of the Fc $\alpha$ Rl:Fc $\alpha$ structures are overlaid onto the two Fc $\alpha$ molecules in the Fc $\alpha-J$ structure to illustrate the potential binding mode between $\mathrm{Fc} \alpha \mathrm{RI}$ and dlgA (only the FcoRI molecules in FcoRl:Fc $\alpha$ are shown for clarity). One side of dlgA is occupied by the J-chain and not exposed for interacting with the $\mathrm{Fc} \alpha \mathrm{RI}$ molecules that are shown in yellow.

\section{MATERIALS AND METHODS}

Protein expression and purification

The DNA fragment encoding IgA1-Fc (residues 241-472) was cloned into a modified pcDNA vector with an N-terminal IL-2 signal peptide followed by a twin-strep tag. The DNA fragments encoding the full-length J-chain and SC were cloned into a modified pcDNA vector with a C-terminal $8 \times$ His tag as previously described. ${ }^{17}$ HEK293F cells were cultured in SMM 293T-I medium (Sino Biological Inc.) at $37^{\circ} \mathrm{C}$, with $5 \% \mathrm{CO}_{2}$ and $55 \%$ humidity. The two plasmids expressing $\operatorname{lgA} 1-\mathrm{Fc}$ and J-chain were co-transfected into the cells using polyethylenimine (Polysciences). Four days after transfection, the conditioned media were collected by centrifugation, concentrated using a Hydrosart Ultrafilter (Sartorius), and exchanged into the binding buffer (25 mM Tris- $\mathrm{HCl}, \mathrm{pH} 7.4,150 \mathrm{mM} \mathrm{NaCl}$ ). The recombinant proteins were isolated using Ni-NTA affinity purification and eluted with the binding buffer supplemented with $500 \mathrm{mM}$ imidazole. The Fca-J complex was further purified using a Superdex 200 increase column (GE Healthcare) and eluted using the binding buffer. SC was expressed and purified as previously described. ${ }^{17}$ To obtain the Fca-J-SC tripartite complex, purified Fca-J and SC were mixed in an 1:2 molar ratio and incubated on ice for $1 \mathrm{~h}$. The complex was then further purified on a Superdex 200 increase column and eluted using the binding buffer.

The DNA fragment encoding S. pneumoniae (strain ATCC33400) $\mathrm{Sps}^{\text {NTD }}$ (residues 38-324) was synthesized by Synbio Technologies and cloned into a modified pQlink vector with an N-terminal $8 \times$ His tag. Sps $A^{\text {NTD }}$ was expressed in BL21(DE3)pLysS E. coli. The E. coli culture was grown in the Luria-Bertani medium at $37^{\circ} \mathrm{C}$ to an $\mathrm{OD}_{600}$ of 0.8 , and then induced with $0.5 \mathrm{mM}$ isopropyl- $\beta-\mathrm{D} 1-$ thiogalactopyranoside at $18{ }^{\circ} \mathrm{C}$ overnight for protein expression. The cells were collected by centrifugation, resuspended in the lysis buffer $(50 \mathrm{mM}$ Tris- $\mathrm{HCl}, \mathrm{pH} 8.0,300 \mathrm{mM} \mathrm{NaCl}, 1 \mathrm{mM}$ phenylmethylsulfonyl fluoride), and then disrupted by sonication. The insoluble debris was removed by centrifugation. The recombinant protein was isolated using Ni-NTA affinity purification following standard procedure and eluted with $50 \mathrm{mM}$ Tris$\mathrm{HCl}, \mathrm{pH}$ 8.0, $300 \mathrm{mM} \mathrm{NaCl}$, and $500 \mathrm{mM}$ imidazole. SpsA ${ }^{\mathrm{NTD}}$ was then further purified by gel filtration chromatography using a Superdex 200 increase column and eluted using the binding buffer. To obtain the Fca-J-SC-SpsA ${ }^{\text {NTD }}$ quadruple complex, purified Fca-J-SC and SpsA ${ }^{\text {NTD }}$ were mixed in a 1:2 molar ratio and incubated on ice for $1 \mathrm{~h}$. The complex was then purified again on a Superdex 200 increase column and eluted using the binding buffer.

\section{Negative staining and cryo-EM}

The samples for EM study were prepared essentially as previously described. ${ }^{17}$ All EM grids were evacuated for 2 min and glowdischarged for $30 \mathrm{~s}$ using a plasma cleaner (Harrick PDC-32G-2). For the negative-staining study, 4- $\mu \mathrm{L}$ aliquots of the Fca-J-SC complex at $0.03 \mathrm{mg} / \mathrm{mL}$ were applied to glow-discharged carboncoated copper grids (Zhong Jing Ke Yi, Beijing). After $\sim 40 \mathrm{~s}$, excessive liquid was removed using a filter paper (Whatman No. 1). The grid was then immediately stained using $2 \%$ uranyl acetate for $10 \mathrm{~s}$ and air dried. The grids were examined on a Tecnai G2 20 Twin electron microscope (FEI) operated at $120 \mathrm{kV}$. Images were recorded using a $4 \mathrm{k} \times 4 \mathrm{k} C \mathrm{CD}$ camera (Eagle, FEI). The Fca-J-SCSps $A^{\text {NTD }}$ sample was stained and examined similarly. To prepare the sample for cryo-EM analyses, $4-\mu \mathrm{L}$ aliquots of Fca-J-SC $(0.3 \mathrm{mg} /$ $\mathrm{mL})$ or Fca-J-SC-SpsA ${ }^{\mathrm{NTD}}(0.2 \mathrm{mg} / \mathrm{mL})$ were applied to glowdischarged holy-carbon gold grids (Quantifoil, R1.2/1.3), blotted with filter paper at $4{ }^{\circ} \mathrm{C}$ and $100 \%$ humidity, and plunged into the liquid ethane using a Vitrobot Mark IV (FEI). Grids screening was performed using a Talos Arctica microscope equipped with Ceta camera (FEI). Data collection was carried out using a Titan Krios electron microscope (FEI) operated at $300 \mathrm{kV}$. Movies were recorded on a K2 Summit direct electron detector (Gatan) in a super resolution mode using the SerialEM software. ${ }^{47} \mathrm{~A}$ nominal magnification of $165,000 \times$ was used, and the exposure rate was 11.668 electrons per $\AA^{2}$ per second. The slit width of the energy filter was set to $20 \mathrm{eV}$. The defocus range was set from -0.8 to $-1.6 \mu \mathrm{m}$. The micrographs were dose-fractioned into 32 frames with a total exposure time of $5.12 \mathrm{~s}$ and a total electron exposure of 60 electrons per $\AA^{2}$. Statistics for data collection are summarized in Supplementary information, Table S1.

\section{Imaging processing}

For 3D reconstruction of the Fca-J-SC complex, a total of 16,264 movie stacks were recorded. Raw movie frames were aligned and averaged into motion-corrected summed images with a pixel size of $0.828 \AA$ by MotionCor $2 .^{48}$ The contrast transfer function (CTF) parameters of each motion-corrected image were estimated by the Gctf program (v1.06). ${ }^{49}$ Relion (v3.07) was used for all the following data processing. ${ }^{50}$ Manual screening was performed to remove lowquality images. A set of 475 particles was manually picked and 
subjected to $2 \mathrm{D}$ classification to generate templates for automatic particle picking. A total of $6,875,153$ particles were then autopicked, which were subjected to another round of 2D classification, resulting in 5,051,275 particles that were kept for the subsequent 3D classifications. Initial model was generated using Relion and used as a reference for 3D classification. Three of the six classes $(665,589$ particles) from the final round of 3D classification were selected and combined for refinement, resulting in a map with a $3.23 \AA$ overall resolution after mask-based post-processing. Finally, Bayesian polishing and CTF refinement were applied, which yielded a density map at a resolution of $3.15 \AA$, based on the gold-standard FSC 0.143 criteria. The local resolution map was analyzed using ResMap $^{51}$ and displayed using UCSF Chimera. ${ }^{52}$ Similar data processing strategies were used for the Fca-J-SC-SpsA complex. The workflows of data processing are illustrated in Supplementary information, Fig. S1.

Model building and structure refinement

The structure of Fca (PDB ID: 10W0), as well as the structures of the J-chain and SC from the FCH-J-SC complex (PDB ID: 6KXS), was docked into the EM map using Phenix ${ }^{53}$ and then manually adjusted using Coot. $^{54}$ The $\beta 5-\beta 6$ hairpin of the J-chain, which is disordered in the FCH-J-SC structure, was built de novo. The $\mathrm{Sps}^{\mathrm{NTD}}$ structure was also built de novo, using the previously determined solution structure of the R2 domain (PDB ID: 1W9R) as a guide. Residues in helices a1-a2 of Sps $A^{\text {NTD }}$ can be unambiguously assigned. The amino acid registrations in helix a3 are not entirely reliable, since this helix is only loosely attached to SC and displays poor densities due to structural flexibility. Refinement was performed using the real-space refinement in Phenix. Figures were prepared with Pymol (Schrödinger) and UCSF Chimera.

\section{StrepTactin pull-down assay}

WT and mutant SC proteins were purified using the Ni-NTA affinity method as previously described. ${ }^{17}$ For the pull-down experiments, they were first incubated with purified Fca-J complex on ice for 1 $\mathrm{h}$. The mixture was then incubated with the StrepTactin beads (Smart Lifesciences) in the binding buffer at $4{ }^{\circ} \mathrm{C}$ for another hour. A twin-strep tag is present on Fca. The beads were spun down and then washed three times with the binding buffer. The bound proteins were eluted off the beads using the binding buffer supplemented with $10 \mathrm{mM}$ desthiobiotin. The results were analyzed by SDS-PAGE and visualized by Coomassie staining.

\section{DATA AVAILABILITY}

The cryo-EM map and atomic coordinates of the Fca-J-SC and Fca-J-SC-SpsA complexes have been deposited in the EMDB (accession codes EMD-30004 and EMD30008) and PDB (accession codes $6 L X 3$ and 6LXW), respectively.

\section{ACKNOWLEDGEMENTS}

We thank the Core Facilities at the School of Life Sciences, Peking University for help with negative-staining EM; the Cryo-EM Platform of Peking University for help with data collection; the High-performance Computing Platform of Peking University for help with computation; the National Center for Protein Sciences at Peking University for assistance with Amersham Imager; and Guilan Li for help with cDNA library. The work was supported by the National Key Research and Development Program of China (2017YFA0505200 and 2016YFC0906000 to J.X.; 2019 YFA0508904 to N.G.), the National Science Foundation of China (31570735 and 31822014 to J.X.; 31725007 and 31630087 to N.G.), the Qidong-SLS Innovation Fund to J.X. and N.G.; and the Clinical Medicine Plus X Project of Peking University to J.X.

\section{AUTHOR CONTRIBUTIONS}

Y.W., Y.L., and Q.Z. performed protein purification and biochemical experiments. Y.W. and G.W. prepared cryo-EM sample and collected data. G.W. and H.S. processed the cryo-EM data, under the supervision of N.G. J.X. built the structural model and wrote the manuscript, with inputs from all authors.

\section{ADDITIONAL INFORMATION}

Supplementary information accompanies this paper at https://doi.org/10.1038/ s41422-020-0336-3.

Competing interests: The authors declare no competing interests.

\section{REFERENCES}

1. Chodirker, W. B. \& Tomasi, T. B. Jr. Gamma-globulins: quantitative relationships in human serum and nonvascular fluids. Science 142, 1080-1081 (1963).

2. Halpern, M. S. \& Koshland, M. E. Noval subunit in secretory lgA. Nature 228, 1276-1278 (1970)

3. Mestecky, J., Zikan, J. \& Butler, W. T. Immunoglobulin M and secretory immunoglobulin A: presence of a common polypeptide chain different from light chains. Science 171, 1163-1165 (1971).

4. Koshland, M. E. The coming of age of the immunoglobulin J chain. Annu. Rev. Immunol. 3, 425-453 (1985).

5. Tomasi, T. B. The discovery of secretory $\lg \mathrm{A}$ and the mucosal immune system. Immunol. Today 13, 416-418 (1992).

6. Woof, J. M. \& Mestecky, J. Mucosal immunoglobulins. Immunol. Rev. 206, 64-82 (2005).

7. Mostov, K. E., Kraehenbuhl, J. P. \& Blobel, G. Receptor-mediated transcellular transport of immunoglobulin: synthesis of secretory component as multiple and larger transmembrane forms. Proc. Natl. Acad. Sci. USA 77, 7257-7261 (1980).

8. Brandtzaeg, P. \& Prydz, H. Direct evidence for an integrated function of J chain and secretory component in epithelial transport of immunoglobulins. Nature 311, 71-73 (1984).

9. Norderhaug, I. N., Johansen, F. E., Schjerven, H. \& Brandtzaeg, P. Regulation of the formation and external transport of secretory immunoglobulins. Crit. Rev. Immunol. 19, 481-508 (1999).

10. Kaetzel, C. S. The polymeric immunoglobulin receptor: bridging innate and adaptive immune responses at mucosal surfaces. Immunol. Rev. 206, 83-99 (2005).

11. Pabst, O., Cerovic, V. \& Hornef, M. Secretory IgA in the coordination of establishment and maintenance of the microbiota. Trends Immunol. 37, 287-296 (2016).

12. Macpherson, A. J., Yilmaz, B., Limenitakis, J. P. \& Ganal-Vonarburg, S. C. IgA function in relation to the intestinal microbiota. Annu. Rev. Immunol. 36, 359-381 (2018).

13. Weiser, J. N., Ferreira, D. M. \& Paton, J. C. Streptococcus pneumoniae: transmission, colonization and invasion. Nat. Rev. Microbiol. 16, 355-367 (2018).

14. Brooks, L. R. K. \& Mias, G. I. Streptococcus pneumoniae's virulence and host immunity: aging, diagnostics, and prevention. Front. Immunol. 9, 1366 (2018).

15. Hammerschmidt, S., Talay, S. R., Brandtzaeg, P. \& Chhatwal, G. S. SpsA, a novel pneumococcal surface protein with specific binding to secretory immunoglobulin A and secretory component. Mol. Microbiol. 25, 1113-1124 (1997).

16. Zhang, J. R. et al. The polymeric immunoglobulin receptor translocates pneumococci across human nasopharyngeal epithelial cells. Cell 102, 827-837 (2000).

17. Li, Y. et al. Structural insights into immunoglobulin M. Science 367, 1014-1017 (2020).

18. Dourmashkin, R. R., Virella, G. \& Parkhouse, R. M. Electron microscopy of human and mouse myeloma serum IgA. J. Mol. Biol. 56, 207-208 (1971).

19. Bonner, A., Furtado, P. B., Almogren, A., Kerr, M. A. \& Perkins, S. J. Implications of the near-planar solution structure of human myeloma dimeric lgA1 for mucosal immunity and IgA nephropathy. J. Immunol. 180, 1008-1018 (2008).

20. Bastian, A., Kratzin, H., Eckart, K. \& Hilschmann, N. Intra- and interchain disulfide bridges of the human $J$ chain in secretory immunoglobulin A. Biol. Chem. Hoppe Seyler 373, 1255-1263 (1992).

21. Frutiger, S., Hughes, G. J., Paquet, N., Luthy, R. \& Jaton, J. C. Disulfide bond assignment in human $\mathrm{J}$ chain and its covalent pairing with immunoglobulin $\mathrm{M}$. Biochemistry 31, 12643-12647 (1992).

22. Frutiger, S., Hughes, G. J., Hanly, W. C., Kingzette, M. \& Jaton, J. C. The aminoterminal domain of rabbit secretory component is responsible for noncovalent binding to immunoglobulin A dimers. J. Biol. Chem. 261, 16673-16681 (1986).

23. Fallgreen-Gebauer, E. et al. The covalent linkage of secretory component to IgA. Structure of slgA. Biol. Chem. Hoppe Seyler 374, 1023-1028 (1993).

24. Coyne, R. S., Siebrecht, M., Peitsch, M. C. \& Casanova, J. E. Mutational analysis of polymeric immunoglobulin receptor/ligand interactions. Evidence for the involvement of multiple complementarity determining region (CDR)-like loops in receptor domain I. J. Biol. Chem. 269, 31620-31625 (1994).

25. Hexham, J. M. et al. A human immunoglobulin (lg)A calpha3 domain motif directs polymeric Ig receptor-mediated secretion. J. Exp. Med. 189, 747-752 (1999).

26. Hamburger, A. E., West, A. P. Jr. \& Bjorkman, P. J. Crystal structure of a polymeric immunoglobulin binding fragment of the human polymeric immunoglobulin receptor. Structure 12, 1925-1935 (2004). 
27. Lewis, M. J., Pleass, R. J., Batten, M. R., Atkin, J. D. \& Woof, J. M. Structural requirements for the interaction of human $\lg A$ with the human polymeric Ig receptor. J. Immunol. 175, 6694-6701 (2005).

28. Stadtmueller B. M. et al. The structure and dynamics of secretory component and its interactions with polymeric immunoglobulins. Elife 5, https://doi.org/10.7554/ eLife.10640 (2016).

29. Herr, A. B., Ballister, E. R. \& Bjorkman, P. J. Insights into IgA-mediated immune responses from the crystal structures of human FcalphaRI and its complex with IgA1-Fc. Nature 423, 614-620 (2003).

30. Ramsland, P. A. et al. Structural basis for evasion of IgA immunity by Staphylococcus aureus revealed in the complex of SSL7 with Fc of human IgA1. Proc. Natl. Acad. Sci. USA 104, 15051-15056 (2007).

31. Chintalacharuvu, K. R. et al. Disulfide bond formation between dimeric immunoglobulin $A$ and the polymeric immunoglobulin receptor during hepatic transcytosis. Hepatology 19, 162-173 (1994).

32. Rosenow, C. et al. Contribution of novel choline-binding proteins to adherence, colonization and immunogenicity of Streptococcus pneumoniae. Mol. Microbiol. 25, 819-829 (1997).

33. Luo, R. et al. Solution structure of choline binding protein $A$, the major adhesin of Streptococcus pneumoniae. EMBO J. 24, 34-43 (2005).

34. Lu, L., Lamm, M. E., Li, H., Corthesy, B. \& Zhang, J. R. The human polymeric immunoglobulin receptor binds to Streptococcus pneumoniae via domains 3 and 4. J. Biol. Chem. 278, 48178-48187 (2003).

35. Elm, C. et al. Ectodomains 3 and 4 of human polymeric Immunoglobulin receptor (hplgR) mediate invasion of Streptococcus pneumoniae into the epithelium. J. Biol. Chem. 279, 6296-6304 (2004).

36. Hammerschmidt, S., Tillig, M. P., Wolff, S., Vaerman, J. P. \& Chhatwal, G. S. Speciesspecific binding of human secretory component to SpsA protein of Streptococcus pneumoniae via a hexapeptide motif. Mol. Microbiol. 36, 726-736 (2000).

37. Kumar, N., Arthur, C. P., Ciferri, C. \& Matsumoto, M. L. Structure of the secretory immunoglobulin A core. Science 367, 1008-1014 (2020).

38. van Egmond, M. et al. IgA and the IgA Fc receptor. Trends Immunol. 22, 205-211 (2001).

39. Monteiro, R. C. \& Van De Winkel, J. G. IgA Fc receptors. Annu. Rev. Immunol. 21, 177-204 (2003)
40. van Spriel, A. B. et al. Mac-1 (CD11b/CD18) is essential for Fc receptor-mediated neutrophil cytotoxicity and immunologic synapse formation. Blood 97 2478-2486 (2001).

41. van Spriel, A. B., Leusen, J. H., Vile, H. \& Van De Winkel, J. G. Mac-1 (CD11b/CD18) as accessory molecule for Fc alpha R (CD89) binding of IgA. J. Immunol. 169, 3831-3836 (2002).

42. Agarwal, S. et al. Human Fc receptor-like 3 inhibits regulatory $T$ cell function and binds secretory IgA. Cell Rep. 30, 1292-1299 (2020).

43. Iannelli, F., Oggioni, M. R. \& Pozzi, G. Allelic variation in the highly polymorphic locus pspC of Streptococcus pneumoniae. Gene 284, 63-71 (2002).

44. Alpan, O., Rudomen, G. \& Matzinger, P. The role of dendritic cells, B cells, and $\mathrm{M}$ cells in gut-oriented immune responses. J. Immunol. 166, 4843-4852 (2001).

45. Rochereau, N. et al. Dectin-1 is essential for reverse transcytosis of glycosylated SIgA-antigen complexes by intestinal M cells. PLoS Biol. 11, e1001658 (2013)

46. Janoff, E. N. et al. Pneumococcal lgA1 protease subverts specific protection by human IgA1. Mucosal. Immunol. 7, 249-256 (2014).

47. Mastronarde, D. N. Automated electron microscope tomography using robust prediction of specimen movements. J. Struct. Biol. 152, 36-51 (2005).

48. Zheng, S. Q. et al. MotionCor2: anisotropic correction of beam-induced motion for improved cryo-electron microscopy. Nat. Methods 14, 331-332 (2017).

49. Zhang, K. Gctf: Real-time CTF determination and correction. J. Struct. Biol. 193 1-12 (2016).

50. Zivanov, J. et al. New tools for automated high-resolution cryo-EM structure determination in RELION-3. Elife 7, https://doi.org/10.7554/eLife.42166 (2018).

51. Kucukelbir, A., Sigworth, F. J. \& Tagare, H. D. Quantifying the local resolution of cryo-EM density maps. Nat. Methods 11, 63-65 (2014).

52. Pettersen, E. F. et al. UCSF Chimera-a visualization system for exploratory research and analysis. J. Comput. Chem. 25, 1605-1612 (2004).

53. Adams, P. D. et al. PHENIX: a comprehensive Python-based system for macromolecular structure solution. Acta Crystallogr. Sect. D: Biol. Crystallogr. 66, 213-221 (2010).

54. Emsley, P., Lohkamp, B., Scott, W. G. \& Cowtan, K. Features and development of Coot. Acta Crystallogr. Sect. D: Biol. Crystallogr. 66, 486-501 (2010). 